\title{
A INFLUÊNCIA DOS RAIOS CÓSMICOS GALÁCTICOS NO CLIMA TERRESTRE
}

\section{THE INFLUENCE OF COSMIC RAYS ON THE TERRESTRIAL CLIMATE}

\section{Williamary Portugal ${ }^{1}$ \\ Alessandra Abe Pacini²}

RESUMO: Este trabalho tem como objetivo apresentar a ideia corrente da comunidade científica acerca das relações entre o Sol, o fluxo de raios-cósmicos galácticos incidentes na Terra e o clima do planeta. Assim, além de uma revisão da literatura, foi feita uma estimativa do impacto da variabilidade solar/raios cósmicos/média de nuvens baixas no clima terrestre, através do balanço radiativo. Os anos de 1996 e 2002, período de mínimo e máximo solar, respectivamente, foram utilizados como referência para a pesquisa. Os resultados indicam que, dentre os forçantes climáticos naturais avaliados, a variação da cobertura de nuvens baixas induzida pela modulação dos raios cósmicos é mais apta a provocar significativas mudanças nas condições climáticas terrestres.

Palavras-chave: relação Sol-Terra; raios cósmicos; mudança climática; climatologia.

ABSTRACT: The objective of this work is to present the current idea of the scientific community about the relation of the Sun, galactic cosmic rays flux incidents on the Earth, and the terrestrial climate. Therefore, in addition to a literature review, an estimate of the impact of variability of solar/cosmic rays/average of low clouds on the terrestrial climate was done, by the radioactive balance. The years of 1996 and 2002, minimum and maximum solar period, respectively, were used as reference for the research. The results showed that, among the evaluated natural climate forces, the variation of low cloud cover induced by cosmic rays modulation is more able to cause important changes on the terrestrial climate conditions.

Keywords: relation Sun-Earth; cosmic rays; climate change; climatology.

\footnotetext{
1 Doutoranda em Física e Astronomia - Universidade do Vale do Paraíba - Univap / Instituto de Pesquisa e Desenvolvimento IP\&D. E-mail: williamary@uol.com.br.

${ }^{2}$ Docente da Univap / IP\&D - Laboratório de Física e Astronomia. E-mail: pacini@univap.br.
} 


\section{INTRODUÇÃO}

A caracterização do clima terrestre/global é altamente complexa e depende tanto de forçantes antrópicos, quanto de forçantes naturais, como a variabilidade da radiação solar incidente na atmosfera terrestre, as erupções vulcânicas, a entrada de partículas no sistema terrestre e as variações geomagnéticas. Em escalas de tempo muito longas, fatores orbitais, como as oscilações periódicas da precessão, obliquidade e excentricidade (ciclos de Milankovitch), justificam as variações climáticas bruscas sofridas pelo planeta entre os glaciais e interglaciais (PILLANS; CHANPPELL; NAISH, 1998). Impressões da atividade magnética solar no clima terrestre, em períodos mais curtos, apontam para três maiores fenômenos como os principais responsáveis por essa relação: o fluxo espectral ultravioleta (UV), a irradiância solar total (IRR), e a modulação dos Raios Cósmicos (RC) (PACINI, 2010).

A Irradiância Solar Total (IRR), considerada como 0 total de energia eletromagnética do Sol, possui uma variação cíclica de 11 anos, com amplitude em torno de $0,1 \%$ (REID, 2000). Porcentagem esta, que não seria capaz de produzir significantes mudanças climáticas no planeta (STOTT; JONES; MITCHELL, 2003). Mas, já as variações em torno de 0,6\%, amplificadas, por exemplo, pelos raios cósmicos (ERMAKOV; STOZHKOV, 2003), poderiam justificar abruptas mudanças na temperatura da Terra, como a que ocorreu durante a pequena era do gelo (LEAN; RIND, 1999).

Originários de supernovas e de outras estrelas supermassivas da Via Láctea, os raios cósmicos galácticos são partículas carregadas com um espectro que varia de

Revista Univap - Edição Especial - revista.univap.br São José dos Campos-SP-Brasil, v. 19, n. 34, nov.2013. ISSN 2237-1753 alguns $\mathrm{MeV}$ até acima de $10^{21} \mathrm{eV}$ de energia (SILVA, 2006). Essas partículas podem influenciar no sistema de absorção e reflexão da radiação solar na atmosfera terrestre (USOSKIN; KOVALTSOV; GENNADY, 2008), pois atuam como os principais agentes ionizantes de compostos voláteis (aerossóis), que se tornam núcleos de condensação, fundamentais para a geração de nuvens e, consequentemente, para o aumento de precipitação. Como o fluxo de raios cósmicos galácticos incidentes na atmosfera terrestre depende inversamente da atividade solar, estando o Sol em período de maior atividade (aumento das manchas solares), a concentração de íons produzidos será menor. O contrário ocorre quando o Sol se encontra no período de menor atividade (diminuição do número de manchas solares).

O planeta Terra tem experimentado períodos de decréscimo e aumento de temperatura ao longo da sua história, com oscilações entre uma série de episódios glaciais e interglaciais, durante os quais as geleiras continentais têm alternadamente avançado e recuado à medida que a temperatura da Terra eleva ou abaixa. Mas, como explicar esses intervalos de variações climáticas? Quais são suas causas e por que ocorrem? Uma explicação pode ser dada em parte por meio da análise do impacto da variabilidade solar/raios cósmicos sobre o balanço radiativo do planeta feita neste trabalho.

\section{METODOLOGIA}

Baseado em dados da literatura sobre o fluxo de RC, de média de nuvens baixas e de IRR (Figuras 1 e 2), caracterizamos um período de mínima e outro de máxima atividade solar, sendo 1996 (Mínimo - IRR = 
1.360,70 W m-2 e RC $=5200 \mathrm{~g} \mathrm{~s}^{-1}$ ) e 2002 (Máximo - IRR $=1.361,80 \mathrm{~W} \mathrm{~m} \mathrm{~m}^{-2} \mathrm{e}$ $\mathrm{RC}=4.800 \mathrm{~g} \mathrm{~s}^{-1}$ ), respectivamente. Com base nesses dados, pôde-se fazer uma primeira estimativa do balanço da radiação solar na superfície da Terra $\left(\tau^{\star}\right)$, em diferentes períodos do ciclo solar. $O \tau^{*}$ representa a quantidade de energia disponível na superfície terrestre, considerando a contribuição da radiação solar que chega perpendicularmente à superfície $\left(K_{1}\right)$ e que, após atravessar a atmosfera tem uma parcela dessa energia refletida de volta para o espaço $\left(\mathrm{K}_{2}\right)$, além do balanço de radiação infravermelha, ondas longas, emitidas pela superfície e pela atmosfera, $I_{1}$ e $I_{2}$ respectivamente, devido às suas temperaturas (Lei de Stefan - Boltzmann, onde a irradiação é proporcional à quarta potência da temperatura - $\left.\mid \alpha T^{4}\right)$.

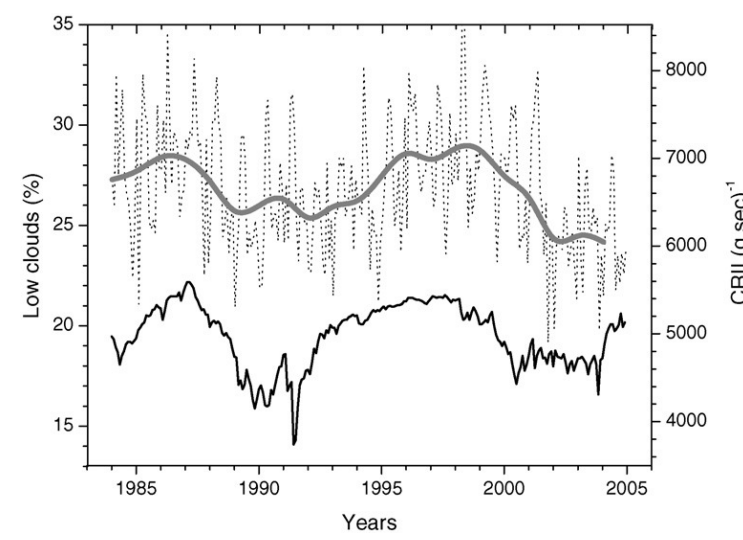

Figura 1 - Variabilidade do número de nuvens baixas (curva superior, eixo da esquerda) e ionização induzida de raios cósmicos entre 3 km de altitude (curva inferior, eixo da direita). As linhas tracejadas na vertical indicam a média anual de nuvens baixas $\left(\sigma_{L}\right)$ e a ionização induzida de raios cósmicos para os anos de 1996 e 2002.

Fonte: USOSKIN; KOVALTSOV; GENNADY, 2008.

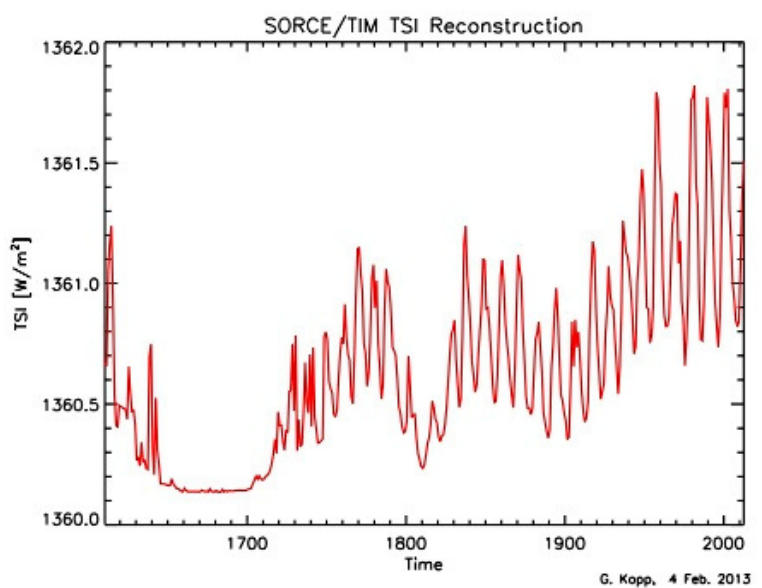

Figura 2 - Reconstrução histórica da Irradiância Solar Total (IRR). As setas indicam os picos referentes ao mínimo e máximo solar, respectivamente para os anos de 1996 e 2002, utilizados nesse trabalho para estimar o balanço de radiação.

Fonte: WANG; LEAN; SHEELEY, 2005.

O balanço radiativo (esquema mostrado pela Figura 3) foi estimado para os anos de 1996 e 2002, utilizando a expressão 1 (STULL, 1995).

$$
\tau *=\mathrm{K}_{1}+\mathrm{K}_{2}+\mathrm{I}_{1}+\mathrm{I}_{2}
$$

sendo $\mathrm{K}_{1}$ e $\mathrm{K}_{2}$ expressos em $\mathrm{W} \mathrm{m}^{-2}$.

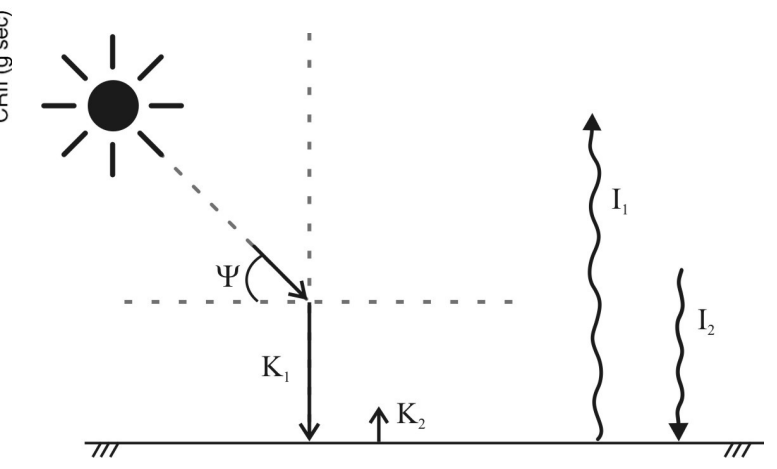

Figura 3 - Desenho esquemático do balanço radiativo.

O cálculo de cada componente é feito, respectivamente, por meio das expressões 2, 3, 4 e 5.

$$
\mathrm{K}_{1}=\mathrm{I} R R \cdot \mathrm{T} \cdot \sin (\Psi)
$$


sendo $\mathrm{T}$ a transmissividade atmosférica (facilidade com que a energia é transportada do topo da atmosfera para a superfície da Terra) e $\Psi$ o ângulo referente à incidência do fluxo solar no topo da superfície (elevação). Neste trabalho, consideramos um fluxo perpendicular à Terra, sendo $\Psi=90^{\circ}$ (região equatorial). $O$ sinal negativo é incorporado na fórmula porque $K_{1}$ representa um fluxo de energia em direção à superfície. A irradiância solar no topo da atmosfera é de, aproximadamente, 1.368,00 $\mathrm{W} \mathrm{m}^{-2} \pm 7$ (valor considerado "constante" na meteorologia chamado de constante solar) (STULL, 1995). Consideramos, em nossos cálculos o valor medido de IRR, mostrado na Figura 3, em cada período simulado. O parâmetro Transmissividade é dado por:

$T=(0,60+0,20 \cdot \sin \Psi) \cdot\left(1-0,40 \sigma_{H}\right) \cdot\left(1-0,70 \sigma_{M}\right)$. $\left(1-0,40 \sigma_{\llcorner}\right)$

onde $\sigma_{H}, \quad \sigma_{M} \quad$ e $\quad \sigma_{L}$ representam, respectivamente, as frações de cobertura de nuvens altas (base $>6.000 \mathrm{~m}$ de altitude), médias (base entre 2.000 e $6000 \mathrm{~m}$ de altitude) e baixas (base $<2.000 \mathrm{~m}$ de altitude). Neste trabalho, as nuvens altas e médias foram desconsideradas, pois, apenas o efeito dos RC na produção de nuvens baixas é encontrado na literatura (Figura 2). De acordo com a Figura 2, para os anos de 1996 e 2002, temos $\sigma_{\mathrm{L}}$, respectivamente, 0,29 e 0,24. Sendo assim, para 1996, temos

$$
\begin{aligned}
& \mathrm{T}=\left(0,60+0,20 \cdot \sin 90^{\circ}\right)(10,40 \cdot 0,29) \\
& \mathrm{T}=0,71 \\
& \mathrm{~K}_{1}=-1360,70 \cdot 0,71 \cdot \sin \left(90^{\circ}\right) \\
& \mathrm{K}_{1(1996)}=-966,10 \mathrm{Wm}^{-2}
\end{aligned}
$$

e para 2002, temos

$$
\begin{aligned}
& \mathrm{T}=\left(0,60+0,20 \cdot \sin 90^{\circ}\right)(10,40 \cdot 0,24) \\
& \mathrm{T}=0,72 \\
& \mathrm{~K}_{1}=-1361,80 \cdot 0,72 \cdot \sin \left(90^{\circ}\right) \\
& \mathrm{K}_{1(2002)}=-980,50 \mathrm{Wm}^{-2}
\end{aligned}
$$

A capacidade de reflexão da superfície é dada pelo parâmetro Albedo (A) e o fluxo de energia que retorna ao espaço pode ser calculado por

$$
\mathrm{K}_{2}=-A \cdot \mathrm{K}_{1}
$$

Neste trabalho, assumimos $A=0,1$ que corresponde ao $A$ da água (STULL, 1995), pois consideramos que a superfície do planeta é coberta $70 \%$ por água. Sendo assim, para 1996, temos

$$
\mathrm{K}_{2}=-0,10 \cdot(-966,10) .
$$

Então,

$$
\mathrm{K}_{2(1996)}=96,61 W m^{-2} .
$$

E, para 2002, temos

$$
\mathrm{K}_{2}=-0,10 \cdot(-980,50) \text {. }
$$

Assim,

$$
\mathrm{K}_{2(2002)}=98,05 W m^{-2}
$$

O balanço do fluxo de ondas longas é dado por

$$
I^{*}=98,50\left(1-0,10 \sigma_{H}-0,30 \sigma_{M}-0,60 \sigma_{L}\right)
$$

onde $I^{*}=I_{1}+I_{2}$. Para 1996, temos $I^{*}(1996)=81,36 \quad$ e para 2002 $I^{*}(2002)=84,32$.

Encontramos, finalmente, um $\tau^{*}$ de $788,13 \mathrm{~W} \mathrm{~m}^{-2}$ para o período de mínimo (ano de 1996) e de $798,13 \mathrm{~W} \mathrm{~m}^{-2}$ para o período de máximo solar (ano de 2002). Outros valores de $\tau^{\star}$ foram obtidos para os mesmos 
períodos, de acordo com as condições especificadas na Tabela 1.

Tabela 1 - Balanço da radiação solar para o ano de 1996 (mínimo solar) e 2002 (máximo solar), considerando as variações de $\sigma_{L}$ e IRR, de acordo com o ciclo solar

\begin{tabular}{|c|c|c|c|}
\hline Condições & 1996 (mínimo) & 2002 (máximo) & $\begin{array}{l}\text { \% Variação } \\
\text { Mín. - Máx. }\end{array}$ \\
\hline $\begin{array}{l}\text { Variando } \sigma_{L} \\
\text { e IRR }\end{array}$ & $\begin{array}{l}\sigma_{\mathrm{L}}=0,29 \\
\mathrm{~S}=1.360,70 \mathrm{~W} \mathrm{~m}^{-2} \\
\tau^{*}=788,13 \mathrm{~W} \mathrm{~m}^{-2} \\
\sigma_{\mathrm{L}}=0,29\end{array}$ & $\begin{array}{l}\sigma_{\mathrm{L}}=0,24 \\
\mathrm{~S}=1.361,80 \mathrm{~W} \mathrm{~m}^{-2} \\
\tau^{*}=798,13 \mathrm{~W} \mathrm{~m}^{-2} \\
\sigma_{\mathrm{L}}=0,24\end{array}$ & $1,3 \%$ \\
\hline $\begin{array}{l}\text { Variando } \sigma_{L} \\
\text { (IRR cte.) }\end{array}$ & $\begin{array}{l}S=1.368,00 \mathrm{~W} \mathrm{~m}^{-2} \\
\tau^{*}=792,79 \mathrm{~W} \mathrm{~m}^{-2} \\
\sigma_{L}=0,27\end{array}$ & $\begin{array}{l}\mathrm{S}=1.368,00 \mathrm{~W} \mathrm{~m}^{-2} \\
\tau^{*}=802,14 \mathrm{~W} \mathrm{~m}^{-2} \\
\sigma_{\mathrm{L}}=0,27\end{array}$ & $1,2 \%$ \\
\hline $\begin{array}{l}\text { Variando IRR } \\
\left.\text { ( } \sigma_{\llcorner} \text {cte. }\right)\end{array}$ & $\begin{array}{l}\mathrm{S}=1.360,70 \mathrm{~W} \mathrm{~m}^{-2} \\
\tau^{\star}=786,95 \mathrm{~W} \mathrm{~m}^{-2}\end{array}$ & $\begin{array}{l}\mathrm{S}=1.361,80 \mathrm{~W} \mathrm{~m}^{-2} \\
\tau^{\star}=787,65 \mathrm{~W} \mathrm{~m}^{-2}\end{array}$ & $0,1 \%$ \\
\hline
\end{tabular}

Analisando os resultados apresentados na Tabela 1, é possível perceber uma taxa de variação do balanço de $\tau^{*}$, do período mínimo para o máximo solar, de $0,1 \%$. Essa variação é exatamente a amplitude da variação cíclica de 11 anos, proposta por Reid (2000). Quando, respectivamente, variam os dois fatores (IRR e $\sigma_{\mathrm{L}}$ ) e apenas $\circ \sigma_{\mathrm{L}}$, mantendo a IRR constante, encontramos as variações de $1,3 \%$ e $1,2 \%$. Esses valores estão acima de $0,6 \%$, o que poderia justificar abruptas mudanças de temperatura da Terra (LEAN; RIND, 1999). Os resultados ainda sugerem que a variação da cobertura de nuvens baixas, induzida pela modulação dos raios cósmicos, é o fator que pode provocar significativas mudanças nas condições climáticas terrestres.

\section{CONCLUSÃO}

Neste trabalho, foi demonstrada uma forte conexão das mudanças do clima terrestre com o restante do universo, por fatores que ultrapassam os limites do planeta. Os resultados mostraram que a variabilidade da atividade solar tem uma contribuição significativa para a mudança do clima da Terra, conjugada a outros fatores, como a ação dos raios cósmicos e a formação de nuvens baixas.

Sendo assim, o conhecimento prévio do período e da intensidade dos mínimos e máximos solares, dos ciclos de 11 anos da atividade do Sol, podem servir como uma importante ferramenta para auxiliar projetos de prevenção e/ou minimização de impactos relacionados a eventos extremos condicionados, em grande parte, pelas bruscas mudanças do clima da Terra.

\section{REFERÊNCIAS BIBLIOGRÁFICAS}

ERMAKOV, V. I.; STOZHKOV, Y. I. Cosmic Rays in the mechanism of thundercloud production. 28th International Cosmic Ray Conferenc. Universal Academy Press, Inc. 4157, 2003.

LEAN, J.; RIND, D. Evaluating Sun-climate relationships since the Little Ice Age. J. Atmosph. Solar-Terr. Phys, v. 61, n. 1-2, p. 25, 1999. 
PACINI, A. A. The role of hidrogen and beryllium isotopes as tracers of solar and climate variability. 2010. Tese (Doutorado em Geofísica Espacial). Instituto de Pesquisas Espaciais, São José dos Campos, 2010.

PILLANS, B.; CHANPPELL, J.; NAISH, T. R. A review of the Milankovitch climatic beat: template for Plio-Pleistocene sea-level changes and sequence stratigraphy. Sedimentary Geology, v. 122, n. 1-4, p.5-21, 1998.

REID, Y. Solar Variability and the Earth's Climate: Introduction and Overview. Space Science Review, v. 94, n. 1-2, p. 1-11, 2000.

SILVA, M. R. Modulação heliosférica ou interplanetária de raios cósmicos e Interação dos raios cósmicos com o campo magnético e a atmosfera terrestres. Exame de
Qualificação de Doutorado. Instituto de Pesquisas Espaciais, São José dos Campos, 2006.

STOTT, P. A.; JONES, G. S.; MITCHELL, J. F. B. Do Models Underestimate the Solar Contribution to Recent Climate Change?. J. Climate, v.16, p. 4079, 2003.

STULL, R. B. Meteorology today for scientists and engineers. Rochester: West Publish, 1995.

USOSKIN, I. G.; KOVALTSOV, G.A.; GENNADY, A. Cosmic rays and climate of the Earth: Possible connection. Comptes Rendus Geoscience, v. 340, p. 441, 2008.

WANG, Y.-M ; LEAN, J. L, SHEELEY, N. R. Jr. Modeling the sun's magnetic field and irradiance since 1713. Astrophysical Journal, v. 625 , p. $522,2005$. 